\title{
Automorphism groups of locally compact connected double loops are locally compact
}

\author{
By
}

RICHARD BÖDI

It is a well-known fact that the group $\Sigma$ of all continuous collineations of a compact projective plane is a locally compact transformation group, see $[4, \S 2],[1, \mathrm{Thm} .1]$, or $[2$, XI.3.1] (all topological groups in this paper are provided with the compact-open topology). This implies that the automorphism group $\Gamma$ of a locally compact connected ternary field is locally compact, since that ternary field coordinatizes some compact projective plane [2, XI.3.2]. For "good" ternary fields, e.g. ternary fields having an associative addition or a diassociative multiplication, the automorphism group $\Gamma$ is even a compact group. However, it is unknown whether the group $\Gamma$ is compact in general. Considering the more general algebraic structure of a double loop instead of a ternary field, it was also an open problem whether the automorphism group of a locally compact connected double loop $\mathscr{D}$ is locally compact. In this paper, we shall give a positive answer to this question. The proof of the following theorem is based on the ideas of the proof in [4], which shows that the automorphism group of a compact connected projective plane is locally compact.

Theorem. The automorphism group of a locally compact connected double loop is a locally compact transformation group with respect to the compact-open topology.

P r o of. Let $\mathscr{D}=(\mathscr{D},+, 0)$ be a locally compact connected double loop and let $\widehat{\mathscr{D}}:=\mathscr{D} \cup\{\infty\}$ denote its one-point compactification. Since $\mathscr{D}$ is metrizable and $\hat{\mathscr{D}}$ is homogeneous by $[2, X I .1 .2]$, the space $\hat{D}$ is also metrizable by the metrization criterion of Urysohn. So let $d$ be a bounded metric for $\widehat{\mathscr{D}}$. The compact-open topology on $\Gamma=\operatorname{Aut}(\mathscr{D})$ is induced by the supremum metric

$$
d^{*}(\gamma, \lambda)=\sup _{x \in \mathscr{Q}} d\left(x^{\gamma}, x^{\hat{\lambda}}\right)
$$

For convenience we may choose the metric $d$ on $\widehat{\mathscr{D}}$ such that $d(0, \infty) \geqq 1$ and $d(0,1) \geqq 1$. For $\varepsilon>0$ and $x_{0} \in \hat{\mathscr{D}}$ we define closed neighborhoods $U_{\varepsilon}\left(x_{0}\right):=\left\{x \in \hat{\mathscr{B}} ; d\left(x, x_{0}\right) \leqq \varepsilon\right\}$ in $\widehat{\mathscr{B}}$ and $U_{\varepsilon}^{*}:=\left\{\gamma \in \Gamma ; d^{*}(\mathbb{1}, \gamma) \leqq \varepsilon\right\}$ in $\Gamma$. In the sequel we shall show that the neighborhood $U_{\varepsilon}^{*}$ is compact if $\varepsilon<1 / 4$. By the Arzela-Ascoli theorem we have to verify the equicontinuity of the set $U_{\varepsilon}^{*}$. 
Assume that the set $U_{\varepsilon}^{*}$ is not equicontinuous for some $\varepsilon<1 / 4$. Then there are sequences $\left(a_{n}\right)_{n \in \mathbb{N}}$ in $\mathscr{D}$ and $\left(\gamma_{n}\right)_{n \in \mathbb{N}}$ in $U_{\varepsilon}^{*}$ such that $\lim _{n \rightarrow \infty} a_{n}=a$ and $d\left(a^{\gamma_{n}}, a_{n}^{\gamma_{n}}\right) \geqq \varepsilon$ hold for all $n \in \mathbb{N}$. Passing to subsequences we may assume by the compactness of $\hat{\mathscr{D}}$ that $\lim _{n \rightarrow \infty} a^{\gamma_{n}}=b \in \hat{\mathscr{D}}$ and $\lim _{n \rightarrow \infty} a_{n}^{\gamma_{n}}=u \in \hat{\mathscr{D}}$. Since the map $x \rightarrow x-b$ is a homeomorphism of

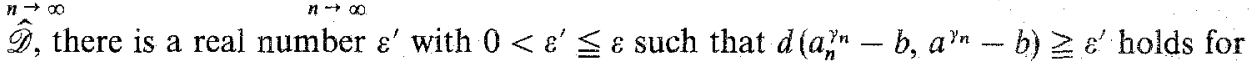
all $n \in \mathbb{N}$. From the inequality

$$
\begin{aligned}
\varepsilon^{\prime} & \leqq d\left(a_{n}^{\gamma_{n}}-b, a^{\gamma_{n}}-b\right) \\
& \leqq d\left(a_{n}^{\gamma_{n}}-b, a_{n}^{\gamma_{n}}-a^{\gamma_{n}}\right)+d\left(a_{n}^{\gamma_{n}}-a^{\gamma_{n}}, 0\right)+d\left(0, a^{\gamma_{n}}-b\right)
\end{aligned}
$$

we obtain that

$$
d\left(\left(a_{n}-a\right)^{\gamma_{n}},(a-a)^{\gamma_{n}}\right)=d\left(a_{n}^{\gamma_{n}}-a^{\gamma_{n}}, 0\right) \geqq \varepsilon^{\prime} / 2
$$

holds for almost all $n \in \mathbb{N}$. By renaming we thus may assume that $\lim a_{n}=0$ and $d\left(a_{n}^{y_{n}}, 0\right) \geqq \varepsilon$ for all $n \in \mathbb{N}$. In particular, we have $\lim _{n \rightarrow \infty} a_{n}^{\gamma_{n}}=u \neq 0$.

Because the double loop $\mathscr{D}$ is separable by [2, XI.1.2], there exists a countable dense subset $M:=\left\{x_{n} ; n \in \mathbb{N}\right\}$ of $\mathscr{D}$ which contains the sequence $\left(a_{n}\right)_{n \in \mathbb{N}}$. Next, we inductively choose for every $m \in \mathbb{N}$ subsequences $\left(\gamma_{m, n}\right)_{n \in \mathbb{N}}$ of $\left(\gamma_{n}\right)_{n \in \mathbb{N}}$ satisfying the conditions

(i) $\lim _{n \rightarrow \infty} x_{m}^{\gamma_{m, n}}=\tilde{x}_{m}$ exists in $\widehat{\mathscr{D}}$,

(ii) $\left(\gamma_{m+1, n}\right)_{n \in \mathbb{N}}$ is a subsequence of $\left(\gamma_{m, n}\right)_{n \in \mathbb{N}}$.

Set $\gamma_{n}^{\prime}:=\gamma_{n, n}=\gamma_{k_{n}}$ and $a_{n}^{\prime}:=a_{k_{n}}$ for all $n \in \mathbb{N}$. Since for the rest of the proof we are only considering the diagonal sequence $\left(\gamma_{n}^{\prime}\right)_{n \in \mathbb{N}}$, no confusion will arise if we now write again $\left(\gamma_{n}\right)_{n \in \mathbb{N}}$ and $\left(a_{n}\right)_{n \in \mathbb{N}}$ instead of $\left(\gamma_{n}^{\prime}\right)_{n \in \mathbb{N}}$ and $\left(a_{n}^{\prime}\right)_{n \in \mathbb{N}}$. Then the equality $\lim _{n \rightarrow \infty} a_{n}^{\gamma_{n}}=u$ still holds and furthermore we have

$$
\lim _{n \rightarrow \infty} x_{m}^{\gamma_{n}}=\tilde{x}_{m} \in \hat{\mathscr{D}}
$$

for any $m \in \mathbb{N}$. Moreover, condition (i) implies that the dense subset $M$ is contained in

$$
L:=\left\{x \in \mathscr{D} ; \lim _{n \rightarrow \infty} x^{\gamma_{n}} \text { exists in } \hat{\mathscr{D}}\right\} \text {. }
$$

In particular, the set $L$ is also dense in $\mathscr{D}$. Consider the map $\lambda: L \rightarrow \hat{\mathscr{D}}: x \mapsto \hat{x}$. Then, for any elements $x, y \in L$ we have

$$
d\left(x, y^{\lambda}\right) \leqq d(x, y)+\varepsilon,
$$

since $d\left(x, y^{\gamma_{n}}\right) \leqq d(x, y)+d\left(y, y^{\gamma_{n}}\right) \leqq d(x, y)+d\left(\mathbb{1}, \gamma_{n}\right) \leqq d(x, y)+\varepsilon$ holds for all $n \in \mathbb{N}$. Set $R:=\left\{x \in L ; \lim _{n \rightarrow \infty} x^{\gamma_{n}} \in \mathscr{D}\right\}$. It can easily be verified that $R$ is a topological double loop with respect to the relative topology. Since all double loop operations in $\mathscr{D}$ are continuous, the closure $\bar{R}$ of $R$ in $\mathscr{D}$ is also a (locally compact) topological double loop. Moreover, the double loop $\bar{R}$ contains the neighborhood $U_{\varepsilon}(0)$. To prove this, note that for any element $y \in L \cap U_{\varepsilon}(0)$ we have $d\left(0, y^{\lambda}\right) \leqq d(0, y)+\varepsilon \leqq 2 \varepsilon<d(0, \infty)$ by inequality (*). This implies that $y^{\lambda} \neq \infty$ and thus we have $y \in R$. Thus the set $R$ is dense in $U_{\varepsilon}(0)$, 
i.e. the closure $\bar{R}$ contains $U_{\varepsilon}(0)$. Consequently, the sub-double-loop $\bar{R}$ is open in $\mathscr{D}$, because it contains an open subset (see $[3,(3.2)]$ ). Since $\mathscr{D}$ is connected, this implies $\bar{R}=\mathscr{D}$.

In the next step we want to show that $u \neq \infty$. For this, note that for almost all $n \in \mathbb{N}$ the inequalities

1) $d\left(a_{n}^{\gamma_{n}}, u\right)<\varepsilon$,

2) $d\left(a_{n}, \infty\right)>1-\varepsilon$

are satisfied. The first inequality follows from the relation $\lim _{n \rightarrow \infty} a_{n}^{\gamma_{n}}=u$ and the second can be deduced from $1 \leqq d(0, \infty) \leqq d\left(0, a_{n}\right)+d\left(a_{n}, \infty\right)$ and $\lim _{n \rightarrow \infty} a_{n}=0$. Combining these two inequalities, we obtain from

$$
d\left(a_{n}, \infty\right) \leqq d\left(a_{n}, a_{n}^{\gamma_{n}}\right)+d\left(a_{n}^{\gamma_{n}}, u\right)+d(u, \infty)
$$

the estimate

$$
d(u, \infty) \geqq(1-\varepsilon)-\varepsilon-\varepsilon=1-3 \varepsilon>0,
$$

which proves that $u \neq \infty$.

Being the pointwise limit of double loop homomorphisms, the restriction $\left.\lambda\right|_{R}: R \rightarrow R^{\lambda}$ is also a double loop homomorphism. Thus the sub-double-loop $R^{\lambda}$ of $\mathscr{D}$ is a topological double loop with respect to the relative topology. Furthermore, the map $\left.\lambda\right|_{R}$ is injective. To see this, fix an element $r \in R \backslash\{0\}$. Since $R$ is a dense subset of $\mathscr{D}$ and since the mapping $x \mapsto r \circ x: R \rightarrow R$ is a bijection of $R$, there is an element $s \in R$ such that $r^{\circ} s \in U_{\varepsilon}(1)$. Thus we obtain from $(*)$

$$
d\left(1,(r \circ s)^{\lambda}\right) \leqq d(1, r \circ s)+\varepsilon \leqq 2 \varepsilon<1 .
$$

Since $d(0,1) \geqq 1$, we infer that $(r \circ s)^{\lambda} \neq 0$. Because $\left.\lambda\right|_{R}$ is a multiplicative map, this means that $r^{\lambda} \neq 0$. Hence, the double loop homomorphism $\left.\lambda\right|_{R}$ is injective. The loop $R$ is infinite, because $R$ is dense in $\mathscr{D}$. So, the set $R^{\lambda}$ is infinite, too, and thus the closure $\overline{R^{\lambda}}$ in $\hat{\mathscr{D}}$ contains an accumulation point $v$ of $R^{\lambda}$. Let $\left(v_{n}\right)_{n \in \mathbb{N}}$ be a sequence in $R^{\lambda}$ which converges to the accumulation point $v$. Then the sequence $\left(v_{n+1}-v_{n}\right)_{n \in \mathbb{N}}$ is also contained in $R^{\lambda}$ and converges to the neutral element 0 . Hence, there is an element $c \in R \backslash\{0\}$ such that the element $b \in R$ which is uniquely determined by the relation $u \circ b=c^{\lambda}$ is not contained in $U_{3 \varepsilon}(\infty)$. Now let $b_{n} \in \mathscr{D}$ denote the (unique) solution of the equation $a_{n} \circ b_{n}=c$. Then we have $\lim _{n \rightarrow \infty} b_{n}=\infty$, because $\lim _{n \rightarrow \infty} a_{n}=0$ and $c \neq 0$. Moreover, we have

$$
c^{\hat{n}}=\lim _{n \rightarrow \infty}\left(a_{n} \circ b_{n}\right)^{\gamma_{n}}=\lim _{n \rightarrow \infty} a_{n}^{\gamma_{n}} \circ b_{n}^{\gamma_{n}} .
$$

Thus the limit $\lim _{n \rightarrow \infty} b_{n}^{\gamma_{n}}$ exists in $\mathscr{D}$, since $\lim _{n \rightarrow \infty} a_{n}^{\gamma_{n}}=u$. Because the element $b$ is the unique solution of the equation $u \circ b=c^{\lambda}$, we consequently obtain that

$$
\lim _{n \rightarrow \infty} b_{n}^{\gamma_{n}}=b \text {. }
$$

So we may choose an integer $n \in \mathbb{N}$ such that $d\left(b_{n}, \infty\right) \leqq \varepsilon$ and $d\left(b, b_{n}^{\gamma_{n}}\right) \leqq \varepsilon$. Inequality (*) now yields the contradiction

$$
3 \varepsilon<d(b, \infty) \leqq d\left(b, b_{n}^{y_{n}}\right)+d\left(b_{n}^{y_{n}}, b_{n}\right)+d\left(b_{n}, \infty\right) \leqq 3 \varepsilon .
$$


This contradiction shows that $U_{\varepsilon}^{*}$ is an equicontinuous set. Hence, the closure $\overline{U_{z}^{*}}$ of $U_{\varepsilon}^{*}$ in the space $C(\widehat{\mathscr{D}}, \widehat{\mathscr{D}})$ of all continuous mapping of $\hat{\mathscr{D}}$ is compact by the Arzela-Ascoli theorem.

To finish the proof, we shall show that $\overline{U_{\varepsilon}^{*}} \subseteq U_{\varepsilon}^{*}$. For that, fix an element $y \in \overline{U_{\varepsilon}^{*}}$. We have to verify that $\gamma$ is a double loop automorphism. Since there is a sequence $\left(\gamma_{n}\right)_{n \in \mathbb{N}}$ in $U_{\varepsilon}^{*}$, which converges to $\gamma$, the map $\gamma$ is a double loop homomorphism. Moreover, the sequence $\left(\gamma_{n}^{-1}\right)_{n \in \mathbb{N}}$ has an accumulation point $\delta$ in the compact set $\overline{U^{*}}$, because $U_{\varepsilon}^{*-1}=U_{\varepsilon}^{*} \subseteq \overline{U_{\varepsilon}^{*}}$. Hence, the sequence $\left(\gamma_{n} \gamma_{n}^{-1}\right)_{n \in \mathbb{N}}$ accumulates at the point $\gamma \delta$. But as $\gamma_{n} \gamma_{n}^{-1}=\mathbb{1}$ holds for each $n \in \mathbb{N}$, this implies that $\gamma \delta=\mathbb{1}$, since composition $C(\hat{D}$, $\widehat{D})$ is continuous. Similarly, the relation $\delta \gamma=\mathbb{1}$ is verified. Consequently, the map $\gamma$ is a bijection and also a double loop automorphism.

\section{References}

[1] T. Grundhörer, Automorphism gtoups of compact projective planes. Geom. Dedicata 21, $291-298$ (1986).

[2] T. GrundhöFER and H. SAlzMANN, Locally compact double loops and ternary fields. In: Quasigroups and Loops: Theory and Applications, O. Chein, H. D. Pflugfelder and J. D. H. Smith, eds. Berlin 1990.

[3] K. H. Hofmann, Topologische Loops. Math. Z. 70, 13-37 (1958):

[4] H. Salzmann, Homogene kompakte projektive Ebenen. Pacific J. Math. 60, 217-233 (1975).

Eingegangen am 25,8.1992

Anschrift des Autors:

Richard Bödi

Mathematisches Institut

Universität Tübingen

Auf der Morgenstelle 10

D-72076 Tübingen 